\title{
Clinicopathological and morphological spectrum of gastrointestinal stromal tumours on multi-detector computed tomography
}

\author{
Rishi Philip Mathew ${ }^{1 \mathrm{E}, \mathrm{F}}$, Joseph Vinod Xavier ${ }^{2 \mathrm{E}, \mathrm{F}}$, Sandeep M. Babukumar ${ }^{2 \mathrm{~A}, \mathrm{~B}}$, Ram Shenoy Bastij ${ }^{2 \mathrm{~A}, \mathrm{C}}$, Hadihally B. Suresh ${ }^{2 \mathrm{~A}, \mathrm{D}}$ \\ ${ }^{1} \mathrm{KG}$ Hospital \& Post Graduate Medical Institute, Coimbatore, India \\ 2Department of Radio-Diagnosis, Father Muller Medical College, Karnataka, India
}

\section{Abstract}

Purpose: To describe the clinicopathological and morphological features of gastrointestinal stromal tumours (GISTs) on multi-detector computed tomography (MDCT).

\begin{abstract}
Material and methods: MDCT (plain and post contrast) images of 25 confirmed cases of GISTs were retrospectively evaluated from our hospital database. The images were analysed for the location, size, growth pattern, attenuation pattern, relation to adjacent structures, presence or absence of ulceration, calcification, metastases, lymphadenopathy, and for any complications such as haemorrhage, intestinal obstruction, etc. Institutional Ethics Committee clearance was obtained prior to the commencement of the study. Statistics used included percentage frequency.

Results: Our study group comprised 14 males and 11 females. The mean age of our study population was 60 years (age range: 40 to 82 years). The mean tumour size was $11.7 \mathrm{~cm}$. The stomach and small bowel accounted for $76 \%$ of the primary tumour site. The commonest imaging appearance of GIST observed in our study was that of an exophytic mass $(76 \%)$ with a heterogenous pattern of enhancement (96\%) with intratumoural necrosis (76\%). MDCT demonstrated complications in three patients (12\%). Six patients presented with metastatic foci (five to the liver and one to the lung), while lymphadenopathy was observed in five patients (20\%). Associated complications included intestinal obstruction (8\%) and retrogastric haematoma (4\%). Incidental findings included uterine fibroid $(n=1)$, ovarian dermoid $(n=1)$, and chronic pancreatitis $(n=1)$.

Conclusions: GISTs are predominantly large tumours with a well circumscribed and exophytic pattern on MDCT, with or without cystic/necrotic areas, and they mostly show a heterogenous pattern of enhancement on post-contrast administration.
\end{abstract}

Key words: gastrointestinal stromal tumors, connective and soft tissue, multi-detector computed tomography.

\section{Introduction}

Gastrointestinal stromal tumours (GISTs) are rare, but they are the most common mesenchymal tumours. GISTs may have their origin anywhere in the gastrointestinal tract (GIT) from the mouth to the rectum, or even in the mesentery or omentum. Ninety per cent of GISTs show a strong immunohistochemical staining for KIT, a tyrosinase kinase growth factor receptor, making them different from other mesenchymal tumours in pathology. Benign
GISTs may be discovered incidentally and are usually excised. Contrast-enhanced multi-detector computed tomography (MDCT) is the most commonly used modality for initial evaluation and staging of GISTs. While 18-FDG positron emission tomography-computed tomography (PET-CT) has emerged as the gold standard for follow-up and evaluation of treatment response. The intention of our study is to evaluate GIST for its anatomical and pathological features on MDCT, as well as to highlight the role of

\section{Correspondence address:}

Dr. Rishi Philip Mathew, KG Hospital \& Post Graduate Medical Institute, 5, Govt. Arts College Road, Coimbatore - 641018, India, e-mail: dr_rishimathew@yahoo.com

Authors' contribution:

A Study design · B Data collection · C Statistical analysis · D Data interpretation · E Manuscript preparation · F Literature search · G Funds collection 
imaging in the detection, characterisation, and treatment response evaluation of GISTs [1].

\section{Material and methods}

MDCT images of 25 patients with GIST confirmed by a combination of histopathology and immunochemistry obtained over a period of four years, from March 2012 to March 2016, were retrospectively evaluated from our hospital database. All the patients underwent MDCT evaluation on a GE Bright Speed 16-Slice MDCT scanner. Initial plain (non-contrast) abdominal images were acquired to look for haemorrhage or calcification. Post-contrast images were obtained after intravenous administration of iopromide (300 mg I/ml, Ultravist ${ }^{\circledR} 300$; Bayer HealthCare Pharmaceuticals, San Francisco, CA) injected at the rate of $3 \mathrm{ml} / \mathrm{s}$ with the help of a Mallinckrodt pressure injector. Arterial and porto-venous phase images were acquired at 18-22 s and 60-65 s, respectively. 3D reconstruction using thin sections $(1 \mathrm{~mm})$ were performed in coronal and sagittal planes for better depiction of the lesion and its extent and involvement of adjacent structures. The images were reviewed on a commercially available workstation.

In those patients who underwent positive contrast CT enterography, patients were kept nil per os for about 4-6 hours prior to ingestion of oral contrast. Positive contrast containing $65 \%$ meglumine diatrizoate $(20 \mathrm{ml}$ dissolved in $1500 \mathrm{ml}$ of water) was orally consumed by the patient. About $1200 \mathrm{ml}$ was consumed over a duration 30-45 minutes, and the remaining $300 \mathrm{ml}$ about 10 minutes prior to commencement of the scan. In order to obtain a uniform distention of the small bowel, patients were required to consume about $150 \mathrm{ml}$ of the contrast mixture every 5 minutes for 50-55 minutes. Imaging was performed about one hour post consumption of the oral contrast mixture.

Detailed clinical and histopathological data were obtained from the hospital database, including patients' demographics such as sex, age, presenting clinical symptoms, etc. The MDCT images were assessed for tumour size, origin, location of the GIT involved by the tumour, tumour attenuation pattern on plain and post contrast administration, presence or absence of ulceration, relationship with adjacent organs and structures, presence or absence of metastases and/or lymphadenopathy, presence of any ancillary findings or complications such as intestinal obstruction, ascites, tumour bowel fistula, intraperitoneal rupture, etc., and for any additional incidental findings.

Institutional Ethics Committee approval was obtained prior to commencement of the study. Statistical analysis involved percentage frequency.

\section{Results}

Of the 25 patients evaluated, 14 were males and 11 were females. The mean age of our study population was 60 years, with the youngest being 40 and oldest being

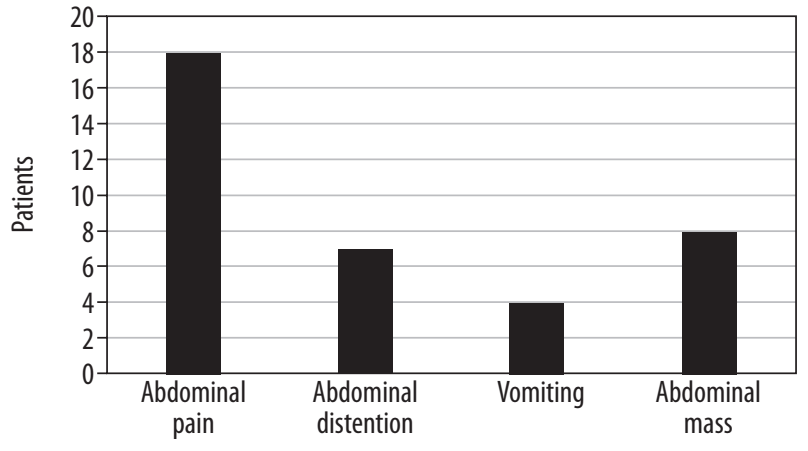

Figure 1. Various clinical symptoms of our study population

Table 1. A summary of the various primary tumour sites of gastrointestinal stromal tumours (GISTs) in the present study vs. other studies

\begin{tabular}{|l|c|c|c|}
\hline Primary site & $\begin{array}{c}\text { Our study } \\
(n=25)\end{array}$ & $\begin{array}{c}\text { Burkill et al. [5] } \\
(n=116)\end{array}$ & $\begin{array}{c}\text { Levy et al. [16] } \\
(n=64)\end{array}$ \\
\hline Oesophagus & 0 & 0 & $1(2 \%)$ \\
\hline Stomach & $7(28 \%)$ & $43(37 \%)$ & $28(44 \%)$ \\
\hline Small Bowel & $12(48 \%)$ & $49(42 \%)$ & $27(42 \%)$ \\
\hline Large Bowel & $2(8 \%)$ & $13(11 \%)$ & $7(11 \%)$ \\
\hline Peritoneum & 0 & 0 & 0 \\
\hline Other/Unknown & $4(16 \%)$ & $11(9 \%)$ & $1(2 \%)$ \\
\hline
\end{tabular}

Others: GISTs arising from omentum, mesentery, retroperitoneum, etc.

Unknown: tumour origin sites that could not be determined on imaging.

82 years old. The most common presenting symptom was abdominal pain (Figure 1). The primary tumour sites have been summarised in Table 1 and compared to two recent studies. The stomach and small bowel accounted for $76 \%$ of the primary tumour location in our study. The mean tumour size was $11.7 \mathrm{~cm}$. Nineteen patients (76\%) presented with an exophytic growth pattern on imaging, while a combination of exophytic-endophytic component (Figure 2) was seen in two cases (8\%), and the remaining two cases presented as wall thickening involving the stomach (Figure 3) and duodenum, respectively. Among the non-gastric GISTs, superior mesenteric artery formed the bulk (45.8\%) of their arterial supply. In six of the cases, the vascular supply could not be identified on MDCT. Except for one case of GIST, which showed minimal enhancement, all the rest, i.e. 24 cases (96\%), showed heterogenous enhancement. Nineteen cases $(76 \%)$ had necrosis (< Hounsfield units), while intratumoural haemorrhage was seen in only one case $(4 \%)$. None of the cases had calcification. Mucosal ulceration was observed in five patients (20\%). At presentation, metastatic foci were seen in six patients; five in the liver (Figure 4) and one in the right lung (Table 2). The secondary liver lesions showed heterogenous enhancement on post contrast administration. Lymphadenopathy (Figure 5) was observed in 10 cases (40\%), mostly in the coeliac and para-aortic region. Ascites (Figure 6) were seen in only eight cases (32\%), while peritoneal deposits were seen in three of our 

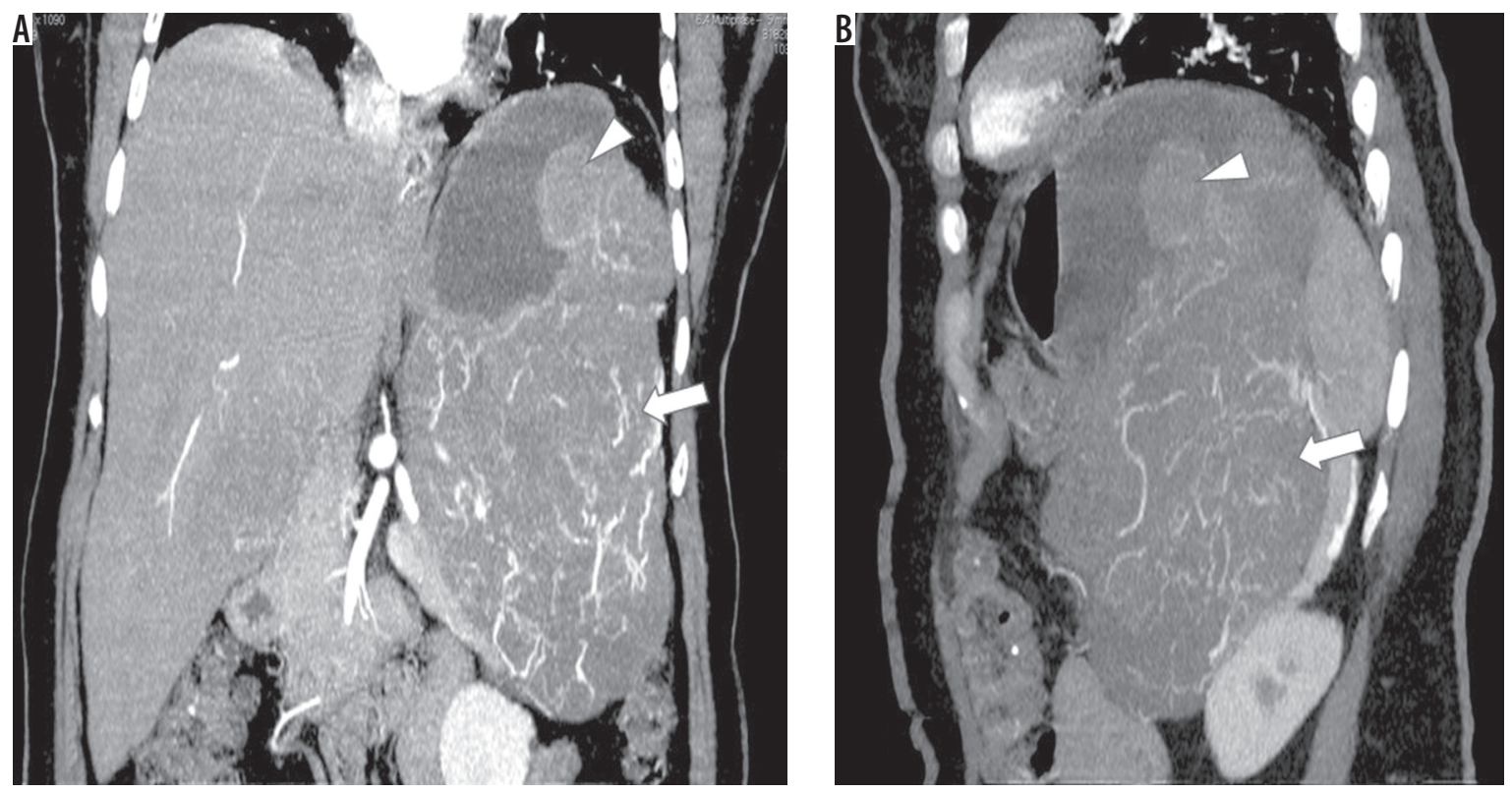

Figure 2. Post-contrast coronal (A) and sagittal (B) reformatted computed tomography images showing a gastric gastrointestinal stromal tumour with an exophytic (arrow) and an endophytic (arrowhead) component
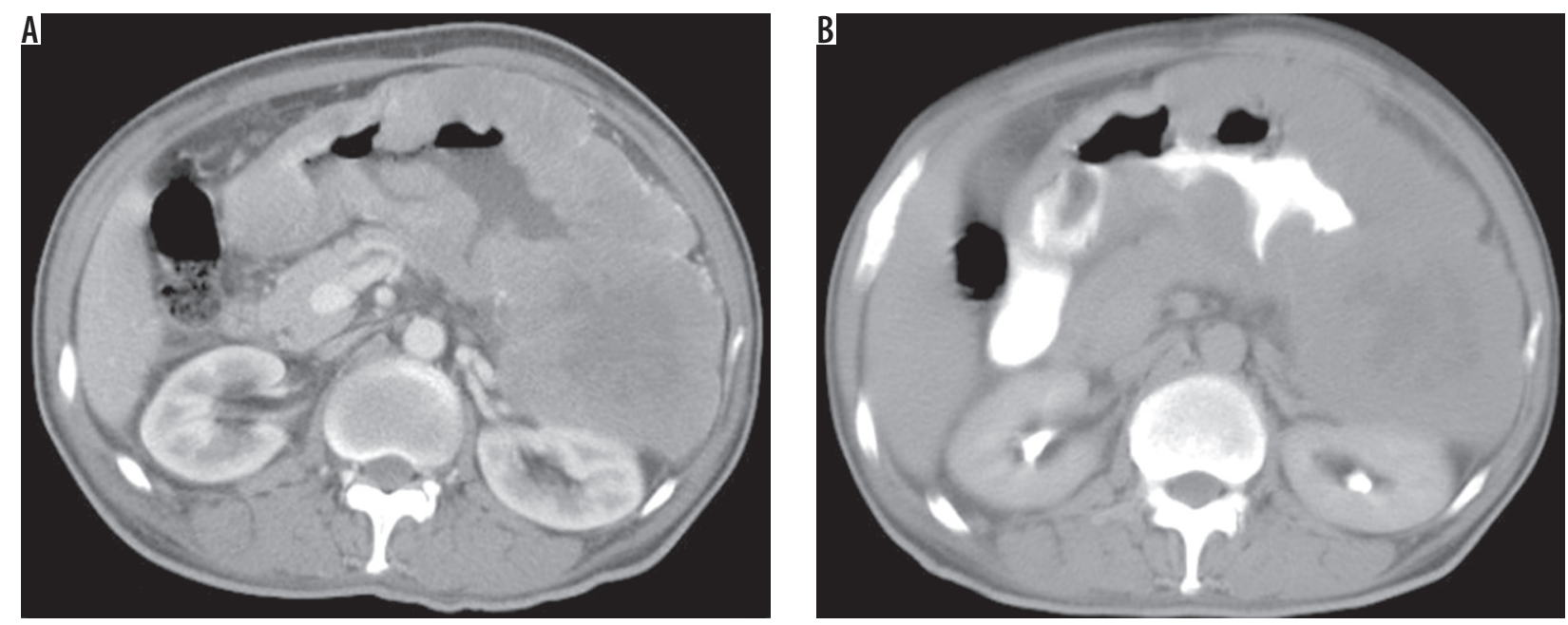

Figure 3. Post contrast axial computed tomography, porto-venous (A), and delayed phase with oral contrast (B) images showing a gastrointestinal stromal tumour presenting as a generalised wall thickening of the stomach

Table 2. Distribution of metastases in our study population

\begin{tabular}{|l|c|}
\hline Site & Number of cases \\
\hline Bone & 0 \\
\hline Liver & 5 \\
\hline Lung & 1 \\
\hline Omentum & 0 \\
\hline
\end{tabular}

cases. Associated complications (Figure 7) in our study included-intestinal obstruction $(n=2,8 \%)$ and retrogastric haematoma $(n=1,4.2 \%)$. Incidental findings in our study included-uterine fibroid $(n=1)$, right ovarian dermoid $(n=1)$, and chronic pancreatitis $(n=1)$. A summary of the various morphological and clinic-pathological spectrum of GISTs in our study population is outlined in Table 3.

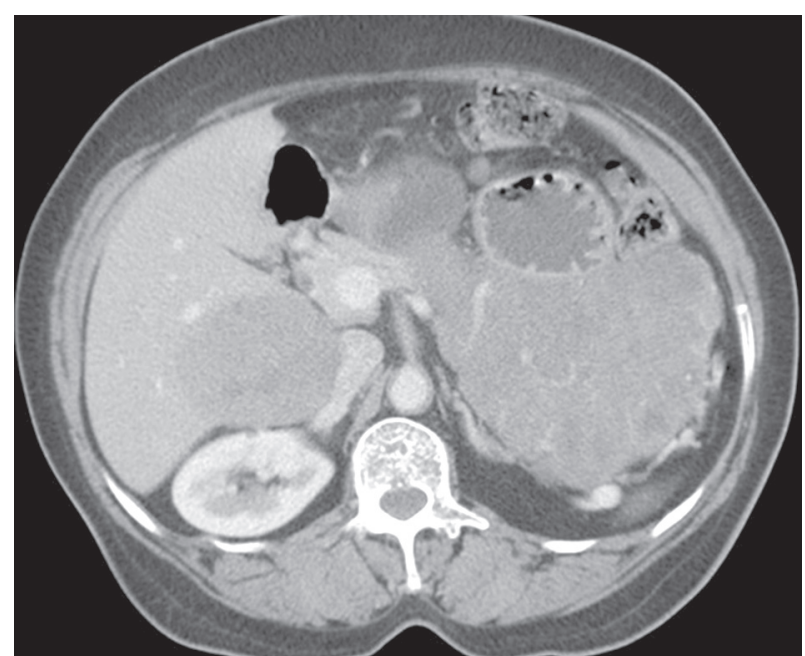

Figure 4. Post-contrast axial computed tomography image of a malignant gastric gastrointestinal stromal tumour in a 60 -year-old female patient with metastases to the liver 

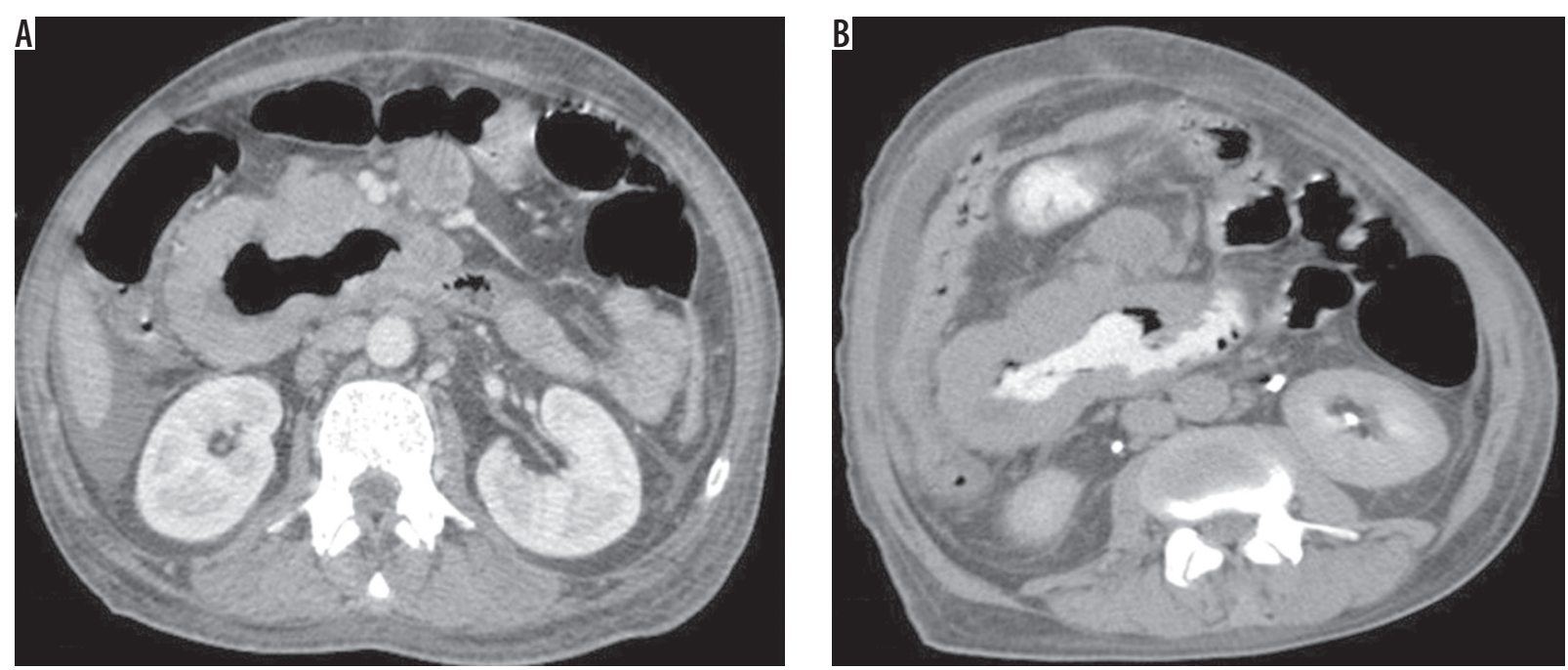

Figure 5. Post contrast axial computed tomography sections, porto-venous (A) and delayed phase with oral contrast (B) images showing a duodenal gastrointestinal stromal tumour with lymphadenopathy
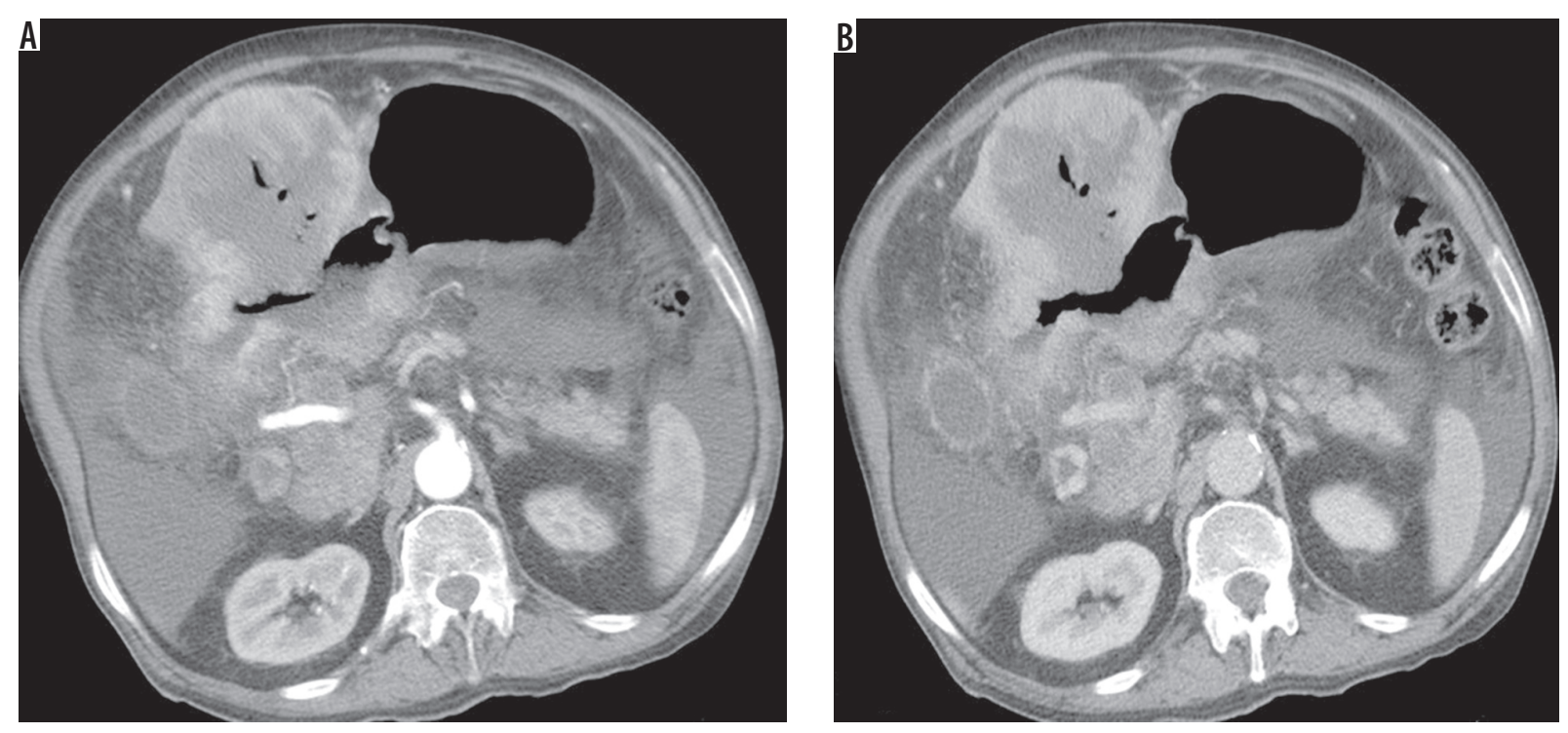

Figure 6. An exophytic gastric gastrointestinal stromal tumour in a 79-year-old male patient with ascites

\section{Discussion}

Gastrointestinal stromal tumours are rare but are the most common mesenchymal tumours [1]. The term GIST was first used by Mazur and Clark in 1983 [2]. These tumours account for $0.1 \%$ to $3 \%$ of all gastrointestinal neoplasms and $5-7 \%$ of sarcomas [3]. These nonepithelial tumours arise from the muscularis propria of the GIT and are believed to originate from the interstitial cells of Cajal - a pluripotent mesenchymal stem cell that acts as a pacemaker for controlling motility. GISTs can occur anywhere along the GIT, i.e. from the oral cavity to the rectum. The most common locations for the tumours are the stomach (60\% of all GISTs), small bowel (30\%), colon and rectum $(5 \%)$, and oesophagus $(<1 \%)$. GISTs may also arise as a primary tumour of the omentum, mesentery, or retroperitoneum. These tumours account for $20 \%$ of all small bowel neoplasms and 0.2 to $1 \%$ of all colorectal tumours, have no sex predilection, and mostly occur in middle-aged and elderly patients [4,5]. Their incidence among children and young adults is rare and is sometimes associated with familial or inherited conditions like neurofibromatosis type-1, Carney triad (gastric GIST, pulmonary chondroma, and paraganglioma), Carney-Stratakis syndrome (gastric GIST and paraganglioma), and familial GIST syndrome. These inherited forms of GIST tend to have a female preponderance, unlike the sporadic variety, which has no sex preponderance [6,7]. During the early stages, patients may be asymptomatic or have minimal symptoms and hence go undetected. However, by the time patients present with symptoms these tumours have grown to a considerable size with spread to other organs [8]. Clinical symptoms mostly depend on the size and site of the lesion, with the most common symptom being bleeding into the bowel or abdominal cavity secondary to ulceration of the tumour mucosa. Patients can 

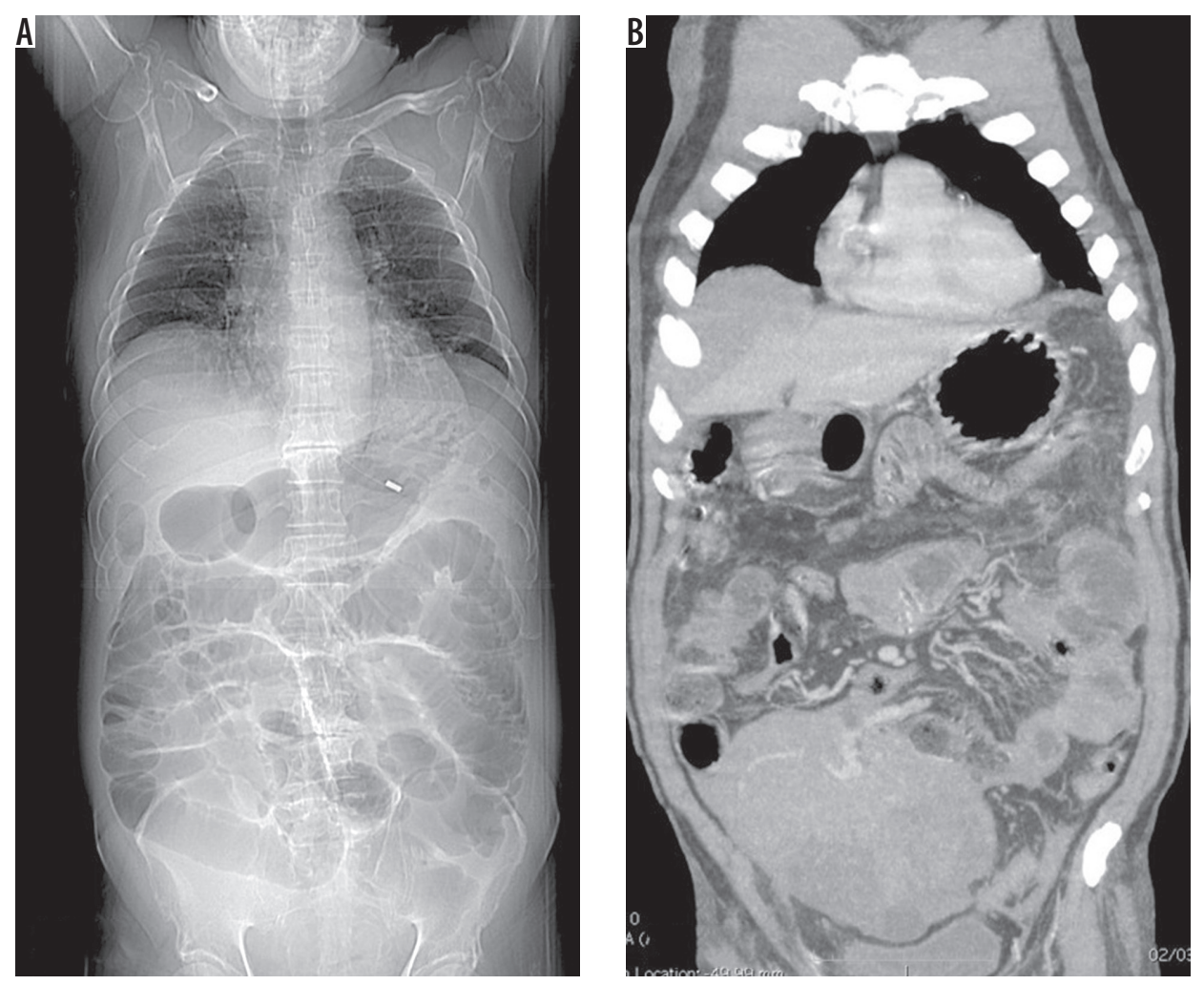

Figure 7. Scout (A) and coronal (B) reformatted post contrast computed tomography images of a 75-year-old male patient with secondary small bowel obstruction due an ileal gastrointestinal stromal tumour

Table 3. A summary of the morphological and clinicopathological spectrum of GIST in our study population

\begin{tabular}{|l|l|}
\hline Characteristics & Findings \\
\hline Males vs. females (years) & 14 vs. 11 \\
\hline Mean age (years) & 60 (range: 40-82) \\
\hline $\begin{array}{l}\text { Mean tumour size (largest } \\
\text { dimension) (cm) }\end{array}$ & 11.4 (range: $3.5-20)$ \\
\hline Symptoms at presentation & $\begin{array}{l}\text { All patients symptomatic } \\
\text { (abdominal pain being } \\
\text { the most common symptom) }\end{array}$ \\
\hline Mean tumour size (cm) & 11.7 \\
\hline $\begin{array}{l}\text { Most common primary } \\
\text { tumour site location }\end{array}$ & Stomach and small bowel $(76 \%)$ \\
\hline Histological subtype & Spindle cell (96\%), epithelioid (4\%) \\
\hline Benign vs. malignant & $19(76 \%)$ vs. $6(24 \%)$ \\
\hline Tumour necrosis & $19 / 25(76 \%)$ \\
\hline Metastases & $6 / 25(24 \%)$ \\
\hline Metastases site & Liver $(n=5)$ and lung $(n=1)$ \\
\hline Lymphadenopathy & $5 / 25(25 \%)$ \\
\hline Ascites & $8 / 25(32 \%)$ \\
\hline Associated complications & $\begin{array}{l}\text { Intestinal obstruction }(n=2) \text { and } \\
\text { retro-gastric haematoma }(n=1)\end{array}$ \\
\hline Incidental findings & $\begin{array}{l}0 \text { varian dermoid }(n=1), \text { pancreatitis } \\
(n=1), \text { uterine fibroid }(n=1)\end{array}$ \\
\hline
\end{tabular}

also present with anaemia due to occult bleeding. Other symptoms include- nausea, vomiting, abdominal pain, abdominal mass, abdominal distention, obstructive jaundice, constipation or diarrhoea, frequent urination, and fatigue. Twenty-five per cent of all GISTs are discovered incidentally during imaging or surgery, and some of them $(\leq 5 \%)$ are found during autopsy $[7,9,10]$.

\section{Genetics}

Histologically GIST can be classified as spindle cell (most common type: 75\%), epithelioid, or pleomorphic mesen-

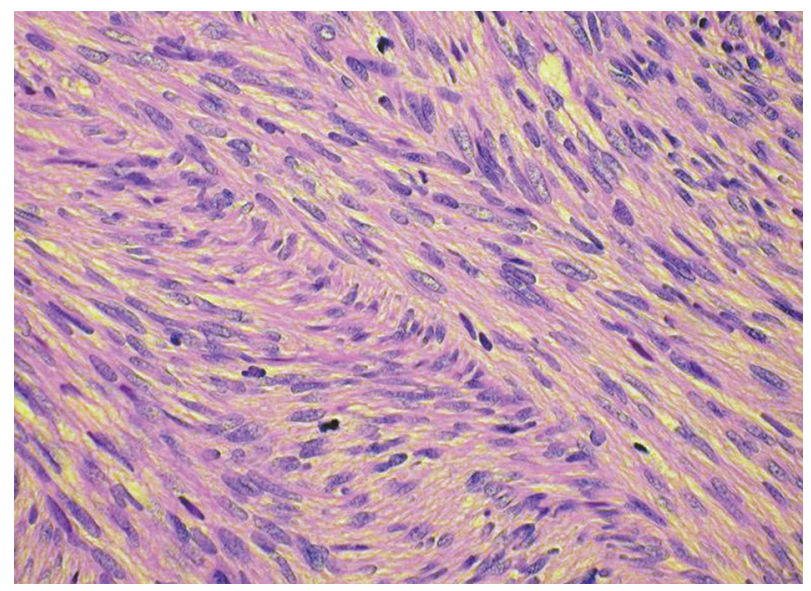

Figure 8. Spindle cells arranged in fascicles and showing mild pleomorphism and mitotic activity 
chymal tumours of the GI tract (Figure 8). All of them express a CD117 stem cell factor receptor called KIT protein, which can be detected immunohistochemically, which forms the key to the diagnosis of a GIST $[1,11]$. KIT is a tyrosine kinase (type 3 ) receptor that helps in the development of melanocytes, germ cells, mast cells, haematopoietic stem cells, interstitial cells of Cajal, and the pacemaker cells of the GI tract that GIST cells most closely resemble. $80-90 \%$ of all GIST show KIT or PDGFRA mutations. Nearly $80 \%$ of patients with GIST tend to show treatment response following administration of imatinib mesylate (Gleevec; Novartis Pharmaceutical Corp.), formerly known as STI-571, which is a targeted KIT tyrosine kinase inhibitor and used for the systemic treatment of GIST [12,13].

In sporadic GISTs, four different areas of KIT mutations have been identified, and in decreasing order of frequency they are: exon 11, exon 9, exon 13, and exon 17. The majority of the KIT mutant proteins are imatinib sensitive. However, exon 17 KIT mutants are primarily resistant, and exon 9 KIT mutants are less sensitive as compared to exon 11 mutants. Gastric GISTs with epithelioid morphology have a strong affinity for PDGFRA mutations, and three different areas of PDGFRA mutations have been found in GISTs. In decreasing order of frequency, they include: exon 18, exon 12, and exon 14. The majority of the PDGFRA mutations occur in exon 18. Some patients who respond to imatinib ultimately develope metastases with drug resistance, secondary to imatinib-resistant mutations in KIT or PDGFRA tyrosine kinase (TK1 and TK2) domains. In recent years, several unique secondary point mutations have been detected and shown to affect the same gene allele as primary mutations [13]. In those patients with a KIT-negative GIST, it is recommended to perform a genotype assay to identify the mutational status of both the KIT and PDGFRA genes in order to establish a definitive diagnosis [14]. Another immunohistochemical marker that may be positive in GIST is S-100, while markers such as alpha smooth muscle antigen ( $\alpha$-SMA) and vimentin are usually negative [15].

The majority of the GISTs are benign (70-80\%). GISTs of the stomach tend to be benign, where they outnumber malignant GISTs by a ratio of 3-5: 1 . Smaller GISTs $(<2 \mathrm{~cm})$ are also considered benign with a low recurrence rate. However, no GIST can be truly labelled as a benign lesion as they tend to have a spectrum of presentation depending on the anatomic site, tumour size, and mitotic frequency [13-15].

\section{Imaging of GISTs}

With advances in treatment resulting in prolonged survival of patients diagnosed with GIST, imaging plays not only a crucial role in the early detection and diagnosis but also for monitoring the treatment and disease progression. On plain radiographs, an incidental soft tissue mass, rarely with calcifications, displacing the gastric air shadow or associated small bowel dilatation may be seen [16].

Barium studies have a limited role in the evaluation of GISTs and may show an intraluminal or submucosal well-circumscribed mass with margins forming an obtuse or right angle with the stomach wall, with the possibility of some luminal irregularity or focal area of ulceration. These lesions may also have a mass effect with or without cavity/fistula formations [17].

On ultrasound, GISTs commonly presents as a large abdominal mass with heterogeneous echotexture with or without necrosis. Often the site of origin may be difficult to determine on ultrasound [18]. A common site for metastases of malignant GIST is the liver, and the lesions are usually hypoechoic compared to the liver parenchyma. Contrast enhanced ultrasonography (CEUS) may be useful for identifying the presence of viable neoplastic tissue in the primary tumour as well as in demonstrating liver metastases [19]. Endoscopic ultrasound is a useful imaging tool for diagnosing small incidental submucosal GISTs, by clearly defining their gut wall layers and allowing biopsy confirmation [20].

Computed tomography is currently the imaging modality of choice both for diagnosis and initial staging of GISTs. CT appearance of GISTs depends on the size, tumour aggressiveness, and time of presentation of the disease. These neoplasms display an exophytic growth pattern because they involve the outer muscular layer and hence the commonest appearance on imaging is that of a lesion arising from the GIT and protruding into the abdominal cavity. Primary GISTs are mostly large, hypervascular, enhancing masses following contrast administration, with a heterogenous appearance secondary to necrosis, haemorrhage, or cystic degeneration. Ulceration and fistulisation are common feature of GITSs, and often tumour vessels may be seen within the mass. The majority of GISTs are well-defined, extraluminal, or intramural masses with nearly $50 \%$ of cases having mucosal ulcerations on the luminal surface $[17,21,22]$. Small GISTs (measuring $<5 \mathrm{~cm}$ ) can be endoluminal and polypoidal in appearance with well-defined, smooth-walled, homogenous soft-tissue lesions involving the gastric wall or the small bowel with an intraluminal component and show homogeneous enhancement after contrast. Large GISTs may be well- or ill-defined, have a heterogenous density on both plain as well as contrast studies with an intraluminal/extraluminal component, displacing/invading adjacent structures. Tumours measuring $>6 \mathrm{~cm}$ commonly demonstrate central necrosis or haemorrhage, while wall calcifications are very rare. After contrast administration, strong peripheral enhancement is seen due to the presence of viable neoplastic tissue $[22,23]$. Large GISTs may also have a 'dumbbell' appearance, due to an intra- and extra-luminal component, or present as extraluminal mass, making identification of its origin extremely difficult. In such situations multiplanar reconstructed images may prove useful $[19,20,24]$. 
Large GISTs due to profound haemorrhage or necrosis can form large cystic masses or cavities, which may communicate with the lumen and contain air, air-fluid levels, or oral contrast medium [25]. About 50\% of all GISTs may metastasise [26]. CT features suggestive of malignancy include: lesions measuring $>5 \mathrm{~cm}$ in diameter, irregular surface, ill-defined margins, invasion of adjacent structures, heterogeneous contrast enhancement, hepatic metastases, and peritoneal dissemination. The most common sites for metastases are the liver and peritoneum, by hematogenous and peritoneal spread, respectively. Rarely, GISTs may metastasise to the soft tissue, lungs and pleura. However, metastases to the lymph nodes are extremely rare compared to gastrointestinal adenocarcinoma. Ascites may be a feature on CT when the tumour has invaded the omentum or peritoneal space $[17,25,27]$. Dual-phase CT is superior to single-phase venous studies because there is a possibility that hypervascular lesions may be missed, as well as for evaluating the disease response to Gleevec therapy and for identifying relapse. Mesenteric metastases are seen better on CT than on magnetic resonance imaging (MRI).

MRI is useful for planning surgery in those patients with a GIST localised to the rectum, for evaluating indeterminate liver lesions on CT and in those patients where $\mathrm{CT}$ is contraindicated. MRI may also be used as an adjunct to CT in the evaluation of large GISTs $[15,28]$. The signal intensity of GISTs on MRI varies greatly and depends on the extent of necrosis and haemorrhage. The solid portions of GISTs are generally hypointense on T1-weighted images (T1-WI), hyperintense on T2-weighted images (T2-WI), and show enhancement after administration of contrast, while areas of haemorrhage within the lesion may appear hypo- or hyper-intense on T1-WI/T2-WI, depending on the age of the bleed [16,29].

Diffusion-weighted imaging (DWI) may be useful to assess response to treatment, which is indicated by an increase in ADC values compared to pre-treatment values and can be seen about one week after initiation of imatinib therapy [30]. Current developments in DWI may also provide new prognostic biomarkers and indicators of response to targeted tumour molecular therapy [31].

18-FDG-PET-CT is the imaging modality of choice for GIST staging, follow-up, as well as for evaluating therapy response [32]. Patients who respond to imatinib demonstrate a marked reduction in the glycolytic metabolism of GISTs which can be identified easily by F18-FDG-PET. This response can be seen one month after initiating therapy, and as early as 24 hours post treatment initiation [33]. 18-FDG PET is also useful when CT findings are ambiguous. For example, patients with a primary GIST with hepatic metastases during imatinib therapy may develop new lesions in the liver. Traditionally, as per the criteria of SWOG (Southwest Oncology Group), the WHO (World Health Organisation), and RECIST (Response Evaluation Criteria in Solid Tumours), these patients would be labelled under disease progression rather than as response to therapy. However, in reality these patients may in fact be responding to treatment. This was observed by Joensuu et al. [34] in the first ever GIST patient treated with imatinib, when the metastatic hepatic lesion in the index patient became hypodense and showed a reduction in contrast enhancement on MRI. Similar findings have since been reported on CT [35]. Another advantage of 18-FDG $\mathrm{PET}$ is in identifying lesions with CT findings suggesting of tumour growth, when in fact the increase in size is due to intratumoural haemorrhage or to tumour swelling unrelated to disease progression. In both the above-mentioned circumstances 18-FDG PET will be negative, confirming the patient's response to treatment [35].

\section{Management of GISTs and recent advances}

Complete surgical resection is the treatment for GISTs $>2 \mathrm{~cm}$ localised to the stomach and for extra-gastric GISTs of any size. Nearly $60 \%$ of all patients with GISTs are cured by surgery [36]. When adjacent organs become involved a multivisceral resection may be needed, such as in the case of duodenal GISTs that may require a pancreatoduodenectomy. However, in such patients, tumour shrinkage by neoadjuvant imatinib treatment should be done in order to allow resection with the possibility of organ preserving surgery [37]. Systemic lymph node dissection is usually not required as these tumours very rarely metastasize to regional lymph nodes [38]. Laparoscopic surgery is best advised for easily accessible lesions and not recommended for lesions $>5 \mathrm{~cm}$ in diameter [39]. Transluminal endoscopic surgery has also been described for small GISTs [40], but the procedure carries the risk of incomplete resection, and as long as techniques for postinterventional gastric wall closure are not standardised, such kinds of surgical procedures must be considered as experimental [41]. As far as adjuvant therapy is concerned, current guidelines of the European Society for Medical Oncology (ESMO) and National Comprehensive Cancer Network (NCCN) state that a minimum of three years of adjuvant imatinib is the standard treatment for patients with a high risk of relapse following primary GIST resection [42]. Novel agents currently under investigation for GIST treatment include: TKIs (e.g. Ponatinib), immunomodulating agents, heat shock protein inhibitors, a phosphoinositide 3-kinase inhibitor, and an insulin-like growth factor 1 inhibitor (e.g. linsitinib) [43,44]. Interventional procedures such as radio-frequency ablation (RFA) [45], transarterial yttrium-90 radio-embolisation [46], and transarterial chemoembolisation (TACE) [47] have all been attempted in patients with GIST metastases, but most of these studies were retrospective and included a limited number of patients.

\section{Differential diagnosis}

Differential diagnosis for GISTs depends on their location. Differentials for GISTs include: leiomyoma, leiomyosar- 
coma, schwannoma, neurofibroma, adenocarcinoma, and carcinoids [48].

\section{Conclusions}

MDCT is the imaging modality of choice for the characterisation and diagnosis of GISTs. Radiologists need to be aware of all the imaging characteristics of GISTs, which may help to identify benign from malignant GISTs. A large exophytic, eccentrically located gastric or bowel wall lesion, which is well circumscribed with or without necrosis and lack of lymphadenopathy, should raise the suspicion of a gastrointestinal stromal tumour. Our study showed that malignant GISTs tend to be larger and have ulcerations. Peritoneal implants and metastases to different organs, especially the liver, and lymphadenopathy, although rare, were additional features of malignant GISTs.

\section{Conflict of interest}

The authors report no conflict of interest.

\section{References}

1. Hasegawa T, Matsuno Y, Shimoda T, et al. Gastrointestinal stromal tumor: consistent CD117 immunostaining for diagnosis, and prognostic classification based on tumor size and MIB-1 grade. Hum Pathol 2002; 33: 669-676.

2. Mazur MT, Clark HB. Gastric stromal tumors, reappraisal of histiogenesis. Am J Surg Pathol 1983; 7: 507-519.

3. Dematteo RP, Lewis JJ, Leung D, et al. Two hundred gastrointestinal stromal tumors; recurrence pattern and prognostic factors for survival. Ann Surg 2000; 231: 51-58.

4. Kim HC, Lee JM, Kim KW, et al. Gastrointestinal stromal tumors of the stomach: CT findings and prediction of malignancy. AJR Am J Roentgenol 2004; 183: 893-898.

5. Burkill GJ, Badran M, Al-Muderis O, et al. Malignant gastrointestinal stromal tumor: distribution, imaging features, and pattern of metastatic spread. Radiology 2003; 226: 527-532.

6. Marrari A, Wagner AJ and Hornick JL. Predictors of response to targeted therapies for gastrointestinal stromal tumors. Arch Pathol Lab Med 2012; 136: 483-489.

7. Joensuu H, Hohenberger P, Corless CL. Gastrointestinal stromal tumour. Lancet 2013; 382: 973-983.

8. Daghter R, Cohen M, Williams G, et al. Imatinib mesylate in the treatment of metastatic and/or unresectable malignant gastrointestinal stromal tumours. Clin Cancer Res 2002; 8: 3034-3038.

9. Behrenwala KA, Spalding D, Wotherspoon A, et al. Small bowel gastrointestinal stromal tumours and ampullary cancer in Type I neurofibromatosis. World J Surg Oncol 2004; 7: 1-4.

10. Caterino S, Lorenzon L, Petrucciani N, et al. Gastrointestinal stromal tumors: correlation between symptoms at presentation, tumor location and prognostic factors in 47 consecutive patients. World J Surg Oncol 2011; 9: 13.

11. Ulusan S, Koç Z. Radiologic findings in malignant gastrointestinal stromal tumors. Diagn Interv Radiol 2009; 15: 121-126.

12. Demetri GD, von Mehren M, Blanke CD, et al. Efficacy and safety of imatinib mesylate in advanced gastrointestinal stromal tumors. N Engl J Med 2002; 347: 47280.

13. Miettinen M, Lasota J. Gastrointestinal stromal tumors: Definition, clinical, histological, immunohistochemical, and molecular genetic features and differential diagnosis. Virchows Arch 2001; 438: 1-12.

14. Demetri GD, Benjamin R, Blanke CD, et al. NCCN task force report: optimal management of patients with gastrointestinal stromal tumors (GIST) - expansion and update of NCCN clinical practice guidelines. J Natl Compr Cancer Netw 2004; 2: S1-S26.

15. Sandrasegaran K, Rajesh A, Rydberg J, et al. Gastrointestinal stromal tumors: clinical, radiologic, and pathologic features. AJR Am J Roentgenol 2005; 184: 803-811.

16. Levy AD, Remotti HE, Thompson WM, et al. Gastrointestinal stromal tumors: radiologic features with pathologic correlation. RadioGraphics 2003; 23: 283-304.

17. Li YZ, Wu PH. Conventional radiological strategy of common gastrointestinal neoplasms. World J Radiol 2015; 7: 7-16.

18. King DM. The radiology of gastrointestinal stromal tumours (GIST). Cancer Imaging 2005; 5: 150-156.

19. Bartolotta TV, Taibbi A, Midiri M, Lagalla R. Focal liver lesions: contrast-enhanced ultrasound. Abdom Imaging 2009; 34: 193209.

20. Kochhar R, Manoharan P, Leahy M, Taylor MB. Imaging in gastrointestinal stromal tumours: current status and future directions. Clin Radiol 2010; 65: 584-592.

21. Suster S. Gastrointestinal stromal tumors. Semin Diagn Pathol 1996; 13: 297-313.

22. Chourmouzi D, Sinakos E, Papalavrentios L, et al. Gastrointestinal stromal tumors: a pictorial review. J Gastrointestin Liver Dis 2009; 18: 379-383.

23. Ghanem N, Altehoefer C, Furtwängler A, et al. Computed tomography in gastrointestinal stromal tumors. Eur Radiol 2003; 13: 16691678.

24. Catalano O, De Lutio di Castelguidone E, Nunziata A, et al. Gastrointestinal stromal tumours: Pictorial review. Radiol Med 2005; 110: 484-491.

25. Lau S, Tam KF, Kam CK, et al. Imaging of gastrointestinal stromal tumour (GIST). Clin Radiol 2004; 59: 487-498.

26. Nilsson B, Bumming P, Meis-Kindblom JM, et al. Gastrointestinal stromal tumors: the incidence, prevalence, clinical course, and prognostication in the preimatinib mesylate era: a population-based study in western Sweden. Cancer 2005; 103: 821-829.

27. Hortan KM, Juluru K, Montogomery E, et al. Computed tomography imaging of gastrointestinal stromal tumors with pathology correlation. J Comput Assist Tomogr 2004; 28: 811-817.

28. Jiang ZX, Zhang SJ, Peng WJ, Yu BH. Rectal gastrointestinal stromal tumors: imaging features with clinical and pathological correlation. World J Gastroenterol 2013; 19: 3108-3116. 
29. Hasegawa S, Semelka RC, Noone TC, et al. Gastric stromal sarcomas: correlation of MR imaging and histopathologic findings in nine patients. Radiology 1998; 208: 591-595.

30. Tang L, Zhang XP, Sun YS, et al. Gastrointestinal stromal tumors treated with imatinib mesylate: apparent diffusion coefficient in the evaluation of therapy response in patients. Radiology 2011;258: 729-738.

31. Zhou HY, Zhang XM, Zeng NL, et al. Use of conventional MR imaging and diffusion-weighted imaging for evaluating the risk grade of gastrointestinal stromal tumors. J Magn Reson Imaging 2012; 36: 1395-1401.

32. Jager PL, Gietema JA, van der Graaf WT. Imatinib mesylate for the treatment of gastrointestinal stromal tumours: best monitored with FDG PET. Nucl Med Commun 2004; 25: 433-438.

33. Van den Abbeele AD, for the GIST Collaborative PET Study Group DanaFarber Cancer Institute, Boston, Massachusetts; OHSU, Portland, Oregon, Helsinki University Central Hospital, Turku University Central Hospital, Finland, Novartis Oncology. F18-FDG-PET provides early evidence of biological response to STI571 in patients with malignant gastrointestinal stromal tumors (GIST). Proc Am Soc Clin Oncol 2001; 20: 362a.

34. Joensuu H, Roberts PJ, Sarlomo-Rikala M, et al. Effect of the tyrosine kinase inhibitor STI571 in a patient with a metastatic gastrointestinal stromal tumor. N Engl J Med 2001; 344: 1052-1056.

35. Choi H, Charnsangavej C, de Castro Faria S, et al. CT evaluation of the response of gastrointestinal stromal tumors after imatinib mesylate treatment: A quantitative analysis correlated with FDG PET findings. AJR Am J Roentgenol 2004; 183: 1619-1628.

36. Joensuu H, Vehtari A, Riihimäki J, et al. Risk of recurrence of gastrointestinal stromal tumour after surgery: an analysis of pooled population-based cohorts. Lancet Oncol 2012; 13: 265-274

37. Rutkowski P, Gronchi A, Hohenberger P, et al. Neoadjuvant imatinib in locally advanced gastrointestinal stromal tumors (GIST): the EORTC STBSG experience. Ann Surg Oncol 2013; 20: 2937-2943.

38. Gervaz P, Huber O, Morel P. Surgical management of gastrointestinal stromal tumours. Br J Surg 2009; 96: 567-578.
39. Lin J, Huang C, Zheng C, et al. Laparoscopic versus open gastric resection for larger than $5 \mathrm{~cm}$ primary gastric gastrointestinal stromal tumors (GIST): a size-matched comparison. Surg Endosc 2014; 28: 2577-2583.

40. Liu B, Song J, Qu B, et al. Endoscopic muscularis dissection for upper gastrointestinal subepithelial tumors originating from the muscularis propria. Surg Endosc 2012; 26: 3141-3148.

41. Schmidt A, Bauder M, Riecken B, et al. Endoscopic full-thickness resection of gastric subepithelial tumors: a single-center series. Endoscopy $2015 ; 47$ : 154-158.

42. ESMO/European Sarcoma Network Working Group: Gastrointestinal stromal tumors: ESMO Clinical Practice Guidelines for diagnosis, treatment and follow-up. Ann Oncol 2014; 25 (Suppl 3): iii21-iii26.

43. Garner AP, Gozgit JM, Anjum R, et al. Ponatinib inhibits polyclonal drug-resistant KIT oncoproteins and shows therapeutic potential in heavily pretreated gastrointestinal stromal tumor (GIST) patients. Clin Cancer Res 2014; 20: 5745-5755.

44. von Mehren M, George S, Heinrich MC, et al. Results of SARC 022, a phase II multicenter study of linsitinib in pediatric and adult wildtype (WT) gastrointestinal stromal tumors (GIST). J Clin Oncol 2014; 32 (Suppl): abstr 10507.

45. Yamanaka T, Takaki H, Nakatsuka A, et al. Radiofrequency ablation for liver metastasis from gastrointestinal stromal tumor. J Vasc Interv Radiol 2013; 24: 341-346.

46. Wright CL, Werner JD, Tran JM, et al. Radiation pneumonitis following yttrium-90 radioembolization: case report and literature review. J Vasc Interv Radiol 2012; 23: 669-674.

47. Takaki H, Litchman T, Covey A, et al. Hepatic artery embolization for liver met astasis of gastrointestinal stromal tumor following imatinib and sunitinib therapy. J Gastrointest Cancer 2014; 45: 494-499.

48. Choi YR, Kim SH, Kim SA, et al. Differentiation of large $(\geq 5 \mathrm{~cm})$ gastrointestinal stromal tumors from benign subepithelial tumors in the stomach: radiologists' performance using CT. Eur J Radiol 2014; $83: 250-260$ 